\title{
Efeitos de altas temperaturas na germinação de sementes de capim-dourado (Syngonanthus nitens (Bong.) Ruhland, Eriocaulaceae): implicações para o manejo
}

Betânia Fichino ${ }^{1,3}$, Alessandra Fidelis ${ }^{1}$, Isabel Schmidt² e Vânia Pivello ${ }^{1}$

Recebido em 3/08/2011. Aceito em 02/04/2012

\begin{abstract}
RESUMO
(Efeitos de altas temperaturas na germinação de sementes de capim-dourado (Syngonanthus nitens (Bong.) Ruhland, Eriocaulaceae): implicações para o manejo). Este estudo teve por objetivo investigar potenciais efeitos do fogo na germinação de sementes de capim-dourado (Syngonanthus nitens) (Bong.) Ruhland (Eriocaulaceae). Sementes coletadas na região do Jalapão, Tocantins, foram submetidas a choques de temperaturas de $60^{\circ}, 100^{\circ} \mathrm{C}, 150{ }^{\circ} \mathrm{C}$ e 200 ${ }^{\circ} \mathrm{C}$ durante 1,3 e 5 minutos. Foram feitas 5 réplicas, com 20 sementes para cada tratamento, e controle. As sementes foram dispostas em placas de Petri e em câmaras de germinação a $28^{\circ} \mathrm{C}$, fotoperíodo $12 \mathrm{~h} / 12 \mathrm{~h}$, por 40 dias. As taxas de germinação das sementes foram analisadas por meio de ANOVA com teste de aleatorização. A maioria dos tratamentos resultou em altas taxas de germinação $(>85 \%)$, exceto $200^{\circ} \mathrm{C} / 3^{\prime}(50 \%)$ e $200^{\circ} \mathrm{C} / 5^{\prime}$, que apresentou uma queda significativa $(4,5 \%, \mathrm{P}<0,05)$. Os resultados obtidos indicam que as sementes de $S$. nitens não são estimuladas nem mortas por altas temperaturas, exceto quando combinados temperatura e tempos de exposição extremos $\left(200^{\circ} \mathrm{C} / 5^{\prime}\right)$. A passagem do fogo é muito rápida durante queimadas nos campos úmidos, onde $S$. nitens ocorre e as temperaturas frequentemente não atingem os $150{ }^{\circ} \mathrm{C}$. Nessas condições, estes resultados indicam que as sementes de $S$. nitens potencialmente sobrevivem à passagem do fogo na maioria das queimadas. Esta informação é de utilidade imediata para o manejo desta espécie de alto valor comercial.
\end{abstract}

Palavras-chave: campo úmido, Cerrado, choque térmico, fogo

\begin{abstract}
(Effects of high temperatures on the germination of Syngonanthus nitens seeds: implications for management. This study aimed to assess the effects of fire on germination of golden-grass (Syngonanthus nitens (Bong.) Ruhland, Eriocaulaceae) seeds. Seeds collected in Jalapão (Tocantins, Brazil) were exposed to heat shock treatments at $60^{\circ} \mathrm{C}, 100^{\circ} \mathrm{C}$, $150^{\circ} \mathrm{C}$ and $200^{\circ} \mathrm{C}$, for 1', 3', and 5. For each treatment and control five replications were used (20 seeds each). Seeds were put on Petri dishes and placed inside germination chambers set at $28^{\circ} \mathrm{C}, 12 \mathrm{~h} / 12 \mathrm{~h}$, for 40 days. Germination rates were analyzed by two-factorial ANOVA, using randomization tests. Most treatments showed high germination rates $(>85 \%)$, except for $200^{\circ} \mathrm{C} / 3^{\prime}(50 \%)$, and $200^{\circ} \mathrm{C} / 5$, which showed germination rates that were significantly lower $(4.5 \%, \mathrm{P}<0.05)$. The results indicated that $S$. nitens seeds were not stimulated or killed by high temperatures, except when an extreme temperature and time of exposure were combined $\left(200^{\circ} \mathrm{C} / 5^{\prime}\right)$. When wet grasslands burn, where $S$. nitens grows, the fire passes very quickly over the area and the temperature during the fire may not exceed $150^{\circ} \mathrm{C}$. Under these conditions, our results suggest that $S$. nitens seeds can potentially survive most fires. These findings are essential for making management recommendations for this species, which has high commercial value.
\end{abstract}

Key words: Cerrado, fire, heat shock, wet grassland

\footnotetext{
${ }^{1}$ Universidade de São Paulo, Departamento de Ecologia, Laboratório de Ecologia da Paisagem e Conservação, São Paulo, SP, Brasil

${ }^{2}$ University of Hawai i, Botany Department, Instituto Brasileiro de Meio Ambiente e Recursos Naturais Renováveis Arizona State University, School of Life Sciences, Tempe, Arizona, U.S.A.

${ }^{3}$ Autor para correspondência: bsfichino@gmail.com
} 


\section{Introdução}

O fogo é um fator determinante para a persistência de diversas espécies no Cerrado brasileiro, sendo comumente utilizado como técnica de manejo em áreas naturais e de agricultura (Alho \& Martins 1995). Além de comumente usado para o manejo da pecuária extensiva, o fogo, na região do Jalapão (Tocantins), é também um importante instrumento de manejo nos campos úmidos, onde ocorre o capim dourado (Syngonanthus nitens (Bong.) Ruhland, Eriocaulaceae). Esta espécie é utilizada na produção artesanal, representando importante fonte de renda. O artesanato é produzido com os escapos florais de $S$. nitens e extrativistas acreditam que a floração dessa espécie é maior um ano após a queima (Schmidt et al. 2007), fazendo com que o manejo tradicional de S.nitens pelas comunidades do Jalapão envolva queimadas regulares, com intervalo de 2 a 3 anos.

Embora o conhecimento tradicional indique que o fogo estimule a floração de $S$. nitens, não há informações sobre os efeitos de altas temperaturas na sobrevivência e germinação de sementes dessa espécie. Estudos anteriores mostraram que, apesar da capacidade de reprodução clonal de S. nitens, a reprodução por sementes é importante para a dinâmica populacional dessa espécie, podendo ser responsável pelo recrutamento de cerca de $40 \%$ dos novos indivíduos (Schmidt et al. 2007). Experimentos que determinem efeitos de altas temperaturas na germinação e viabilidade de suas sementes podem, portanto, contribuir significativamente para o manejo dessa espécie economicamente importante. A determinação dos efeitos das altas temperaturas e diferentes tempos de exposição sobre as sementes de S. nitens permitirá fornecer indicadores para um melhor uso do fogo como ferramenta para seu manejo, de forma a equilibrar a manutenção de suas populações nos campos úmidos, em longo prazo, e sua utilização pela população local.

Sendo assim, este estudo visou investigar a influência do fogo na germinação de $S$. nitens, por meio de tratamentos de choque térmico, buscando melhor compreender a biologia dessa espécie. Especificamente, buscamos responder as seguintes questões: a) a exposição de sementes de $S$. nitens a altas temperaturas (entre $60^{\circ} \mathrm{C}$ e $200^{\circ} \mathrm{C}$ ) inibe ou estimula a sua germinação? b) como interagem diferentes tempos de exposição (1, 3 e 5 minutos) e altas temperaturas (60 ${ }^{\circ} \mathrm{C}, 100{ }^{\circ} \mathrm{C}, 150{ }^{\circ} \mathrm{C}$ e $200{ }^{\circ} \mathrm{C}$ ) nas taxas de germinação das sementes dessa espécie? c) qual a viabilidade das sementes após um ano de colheita?

\section{Material e métodos}

\section{Espécie estudada}

S. nitens é uma sempre-viva bastante comum e endêmica do Brasil Central, sendo característica de campos úmidos adjacentes a veredas e a matas de galeria inundáveis (Giulietti et al. 1988). A espécie é policárpica, podendo atingir maturidade sexual no primeiro ano de vida. Cada planta é formada por uma roseta basal de folhas com, em média, 4 centímetros de diâmetro, que pode produzir 1 a 2 escapos anualmente. As rosetas podem se reproduzir clonalmente, por gemas laterais. Na região do Jalapão, a produção de escapos inicia-se entre abril e maio, e a floração a partir de julho. Cada escapo produz uma flor em forma de capítulo e, após polinização pelo vento, cada capítulo produz, em média, 60 sementes, de 0,7 a 1,0 mm, a partir de agosto. A dispersão ocorre pelo vento, majoritariamente após meados de outubro (Schmidt et al. 2007).

Como o fogo representa uma ferramenta de manejo para estimular a floração de $S$. nitens, ele é utilizado tradicionalmente pelas comunidades locais, que queimam os campos úmidos a cada 2 ou 3 anos, na estação seca (maio a outubro, Schmidt et al. 2007).

\section{Região de estudo}

As sementes de $S$. nitens foram coletadas no Parque Estadual do Jalapão (PEJ) e na Área de Proteção Ambiental Jalapão (APA Jalapão) $\left(10-12^{\circ} \mathrm{S}\right.$ e $\left.45-47^{\circ} \mathrm{W}\right)$, que se encontram inseridos na bacia hidrográfica dos rios Araguaia e Tocantins (MMA 2002), no estado de Tocantins. A precipitação anual média na região é de $1.700 \mathrm{~mm}$ ( $90 \%$ de outubro a abril) e a média das temperaturas é de $27^{\circ} \mathrm{C}$ (SEPLAN 2003). A vegetação predominante no PEJ é de formações campestres (campo sujo, campo limpo e campo cerrado), com presença de florestas de galeria e veredas, dominadas por buritis (Mauritia flexuosa). Ao redor dessas florestas e veredas, sobre organossolos, encontram-se os campos úmidos, dominados por gramíneas e espécies das famílias Xyridaceae, Cyperaceae e Eriocaulaceae e, dentre estas, $S$. nitens, o capim-dourado (Schmidt et al. 2007).

\section{Metodologia de estudo}

Sementes de $S$. nitens foram coletadas de diferentes indivíduos em cinco áreas de campo úmido, em setembro de 2009, proporcionando desta maneira, uma maior variabilidade genética. Elas foram armazenadas em sacos de papel, sob temperatura ambiente, para posterior separação em laboratório.

Para o experimento com choque térmico, foram utilizadas cinco réplicas por tratamento, com 20 sementes cada. As sementes foram colocadas em forno pré-aquecido, com temperatura e tempo de exposição controlados. A fim de se evitar pseudo-réplicas, cada réplica sofreu o choque separadamente, com temperatura e tempo de exposição adequados (Morrison \& Morris 2000). Além do controle, que não foi exposto ao aquecimento, as sementes testadas foram expostas às temperaturas de $60^{\circ} \mathrm{C}, 100^{\circ} \mathrm{C}, 150^{\circ} \mathrm{C}$ e $200^{\circ} \mathrm{C}$, em tempos de exposição de 1,3 e 5 minutos, totalizando 12 tratamentos.

Após os tratamentos, as sementes foram colocadas em placas de Petri, com papel de filtro previamente esterilizado e embebido em água. As amostras foram colocadas para 
germinar em câmaras de germinação com regime de luz controlado $(12 \mathrm{~h} / 12 \mathrm{~h})$ e temperatura constante de $28^{\circ} \mathrm{C}$, durante 40 dias. Nesse período, foram feitas observações a cada três dias para verificar o número de sementes germinadas. As sementes foram consideradas germinadas quando se deu o surgimento da radícula ou cotilédone.

As diferenças das taxas de germinação $(G)$ entre os tratamentos foram testadas por meio de análises de variância com testes de aleatorização, com distância Euclidiana como medida de semelhança (10.000 iterações), considerando-se dois fatores: tempo e temperatura. Utilizou-se testes de aleatorização porque eles não partem da premissa de distribuição normal dos dados (Manly 2007). Todas as análises estatísticas foram feitas com o software MULTIV (Pillar 2005).

Um ano após a coleta, 100 sementes armazenadas em sacos de papel foram submetidos ao teste de viabilidade usando solução de 1,3,5 trifenil tetrazólio com concentração de $1 \%$, incubando-as no escuro a $30^{\circ} \mathrm{C}$ durante $24 \mathrm{~h}$, e analisado visualmente quanto ao padrão de coloração. A visualização pôde ser feita por transparência das sementes.

\section{Resultados e discussão}

Syngonanthus nitens mostrou altas taxas de germinação ( $\mathrm{G}>85 \%$ na maioria dos tratamentos), a qual iniciou a partir do terceiro dia no germinador. Mais de $70 \%$ das sementes germinaram durante os 10 primeiros dias, resultando em um rápido aumento do número de sementes germinadas até o $15^{\circ}$ dia. A partir do $35^{\circ}$ dia, mais de $80 \%$ das sementes já haviam germinado (Fig.1). Portanto, a viabilidade das sementes de Syngonanthus nitens é superior a $80 \%$. O comportamento germinativo de $S$. nitens neste experimento foi semelhante ao encontrado para esta espécie em experimentos anteriores, que atingira taxas de germinação de $92 \%$ (Schmidt et al. 2007).

A germinação de sementes de $S$, nitens não foi alterada pela exposição a altas temperaturas por curtos períodos. A taxa de germinação foi superior a $85 \%$, exceto para os tratamentos de $200{ }^{\circ} \mathrm{C}$ por 3 minutos $(\mathrm{G}=50 \%)$ e a $200{ }^{\circ} \mathrm{C}$ durante 5 minutos, no qual houve significativa queda nas taxas de germinação ( $\mathrm{G}=4.5 \%$, $\mathrm{p}=0,009$, Fig.2).

A resistência a altas temperaturas indica que sementes de S. nitens podem sobreviver e germinar após a passagem do fogo. Experimentos em campos úmidos do Jalapão indicam que o tempo em que a vegetação permanece sob altas temperaturas é curto ( $<60$ segundos) e que, mesmo durante a passagem do fogo, as temperaturas de alguns lugares pode não ultrapassar os $200^{\circ} \mathrm{C}$ (Schmidt 2011).

Os resultados encontrados também corroboram as observações de Hanley et al. (2003), que observou a existência de relação entre o tamanho das sementes e sua resistência a altas temperaturas. Segundo estes autores, espécies com sementes pequenas, como é o caso de $S$. nitens, são mais resistentes a altas temperaturas do que espécies com se-

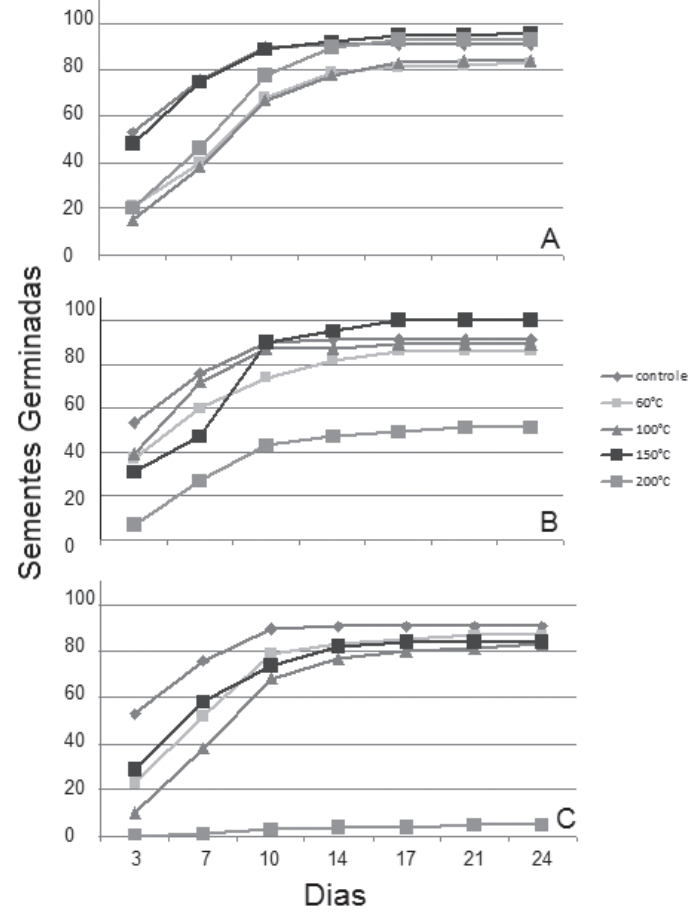

Figura 1. Número de sementes de $S$. nitens germinadas ao longo do experimento: A - 1 minuto; B - 3 minutos ; C - 5 minutos ( $\mathrm{N}=100 /$ tratamento).
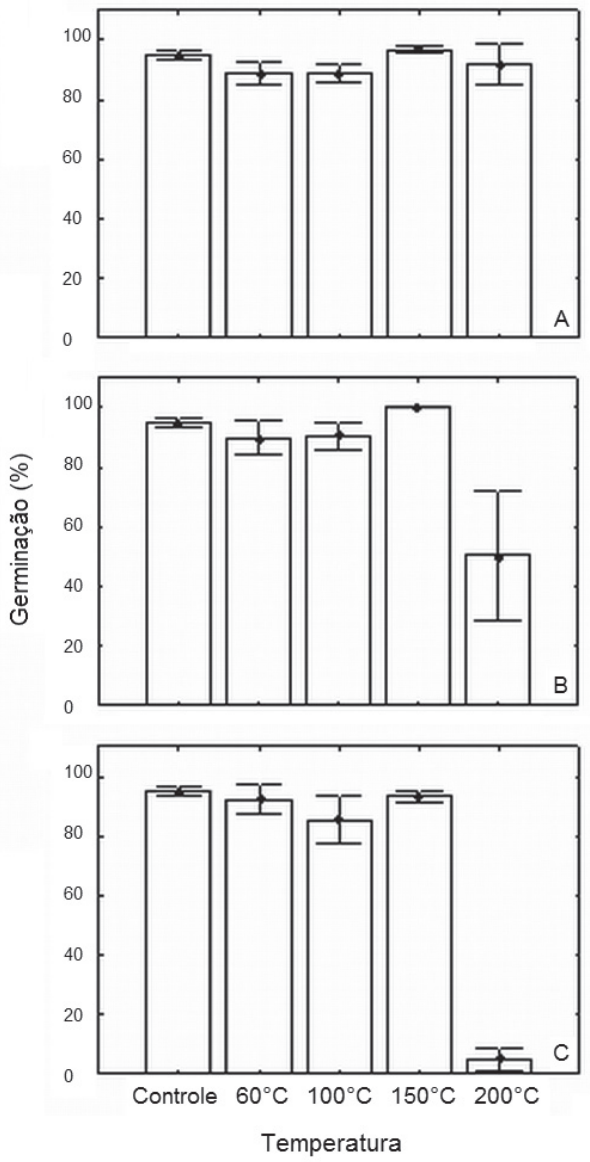

Figura 2. Porcentagem de sementes de S. nitens germinadas após 40 dias nas diferentes temperaturas e tempos de exposição: A - 1 minuto; B - 3 minutos; C - 5 minutos. Barras são as médias e linhas de erro representam o erro padrão $( \pm 1)$. 
mentes grandes. Como sementes pequenas, ao contrário das grandes, mantém-se próximas ou sobre a superfície do solo para germinarem, sua maior resistência a altas temperaturas, em regiões periodicamente submetidas ao fogo, constitui uma adaptação a esse fator ambiental.

O teste de viabilidade mostrou que, após um ano, menos de $5 \%$ das sementes ainda estavam viáveis, o que indica que a espécie não cria um banco de sementes de longa duração.

Estes resultados são extremamente úteis e, através do uso dessas informações para o desenvolvimento de técnicas de manejo coerentes com a relação dessa espécie e o uso do fogo, podem contribuir para o desenvolvimento de recomendações de manejo sustentável de $S$. nitens e dos campos úmidos do Jalapão e outras regiões do Cerrado em que esta espécie tem importância econômica.

\section{Conclusões}

O fogo é utilizado como ferramenta de manejo em campos úmidos para estimulação da produção de escapos florais de Syngonanthus nitens. O fogo nessas áreas é rápido e geralmente as temperaturas altas ocorrem por menos de um minuto. Os resultados destes trabalho indicam que a passagem de fogo pode não prejudicar a sobrevivência e germinação de sementes desta espécie de relevante importância econômica em seu ambiente natural, visto que houve inibição da germinação de sementes somente após exposição destas a $200^{\circ} \mathrm{C}$ por 5 minutos.

\section{Agradecimentos}

As autoras agradecem ao Mauricio Perine pelo auxilio nos experimentos em laboratório e o Dr Sérgio Tadeu Meirelles pelo uso das câmaras de germinação. Além disso, as autoras também agradecem a PEQUI e ao Nílton Ferreira Barborsa pelo apoio técnico, a NATURATINS e funcionários do Parque Estadual do Jalapão, pelas autorizações de coleta e de pesquisa. Este projeto foi financiado pela FAPESP (2008/10049-9).

\section{Referências Bibliográficas}

Alho, C.J.R. \& Martins, E.S. 1995. De grão em grão, o cerrado perde espaço. Documento para discussão. Brasil. WWF/ PRO-CER.

Giulietti, N.; Giulietti, A.M.; Pirani, J.R. \& Menezes, N.L. 1988. Estudos em sempre-vivas: importância econômica do extrativismo em Minas Gerais. Acta Botanica Brasilica 1: 179-193.

Hanley, M.E.; Unna, J.E. \& Darvill, B. 2003. Seed size and germination response: a relationship for fire-following plant species exposed to thermal shock. Oecologia 134: 18-22.

Manly, B.F.J. 2007. Randomization, bootstrap, and monte carlo methods in biology. Boca Raron, Chapman \& Hall/CRC.

Miranda, A.C.; Miranda, H.S.; Dias, I.F.O. \& Dias, B.F.S. 1993. Soil and air temperatures during prescribed cerrado fires in Central Brazil. Journal of Tropical Ecology 9: 313-320.

Morrison, D.A. \& Morris, E.C. 2000. Pseudoreplication in experimental designs for the manipulation of seed germination treatments. Austral Ecology 25: 292-296.

Pillar, V.D. 2005. Multiv - Multivariate Exploratory Analysis, Randomization Tests and Bootstraps Resampling. Universidade Federal do Rio Grande do Sul, Brasil.

Pivello, V.R. \& Norton, G.A. 1996. Firetool: An expert system for the use of prescribed fires in cerrado (Brazlian savanna) conservation areas. Journal of Applied Ecology 33: 348-356.

Schmidt, I.B.; Figueiredo, I.B. \& Scariot, A. 2007. Ethnobotany and Effects of Harvesting on the Population Ecology of Syngonanthus nitens (Bong) Ruhland (Eriocaulaceae), a NTFP from Jalapão Region, Central Brazil. Economic Botany 61: 73-85.

Schmidt, I.B. 2011. Effects of local ecological knowledge, harvest and fire on golden-grass (Syngonanthus nitens, Eriocaulaceae), a nontimber forest product (NTFP) species from the Brazilian savanna. Botany Department / Ecology, Evolution and Conservation Biology program. Honolulu, University of Hawai'i at Manoa.

SEPLAN - Secretaria do Planejamento e Meio Ambiente, 2003. Plano de Manejo do Parque Estadual do Jalapão, Palmas, TO. Disponível em: www.seplan.to.gov.br 